\title{
Particle Motion in Monopoles and Geodesics on Cones
}

Maxence MAYRAND

Department of Mathematics and Statistics, McGill University,

805 Sherbrooke Street West, Montreal, Quebec, Canada, H3A 0B9

E-mail: maxence.mayrand@mail.mcgill.ca

Received July 31, 2014, in final form November 01, 2014; Published online November 04, 2014

http://dx.doi.org/10.3842/SIGMA.2014.102

\begin{abstract}
The equations of motion of a charged particle in the field of Yang's SU(2) monopole in 5-dimensional Euclidean space are derived by applying the Kaluza-Klein formalism to the principal bundle $\mathbb{R}^{8} \backslash\{0\} \rightarrow \mathbb{R}^{5} \backslash\{0\}$ obtained by radially extending the Hopf fibration $S^{7} \rightarrow S^{4}$, and solved by elementary methods. The main result is that for every particle trajectory $\mathbf{r}: I \rightarrow \mathbb{R}^{5} \backslash\{0\}$, there is a 4-dimensional cone with vertex at the origin on which $\mathbf{r}$ is a geodesic. We give an explicit expression of the cone for any initial conditions.
\end{abstract}

Key words: particle motion; monopoles; geodesics; cones

2010 Mathematics Subject Classification: 70H06; 34A26; 53B50

\section{Introduction}

The problem of the classical motion of an electrically charged particle in the field of Dirac's magnetic monopole is a system of three second-order non-linear differential equations, written concisely as

$$
\ddot{\mathbf{r}}=\lambda \frac{\mathbf{r} \times \dot{\mathbf{r}}}{|\mathbf{r}|^{3}}
$$

for $\mathbf{r} \in \dot{\mathbb{R}}^{3}:=\mathbb{R}^{3} \backslash\{0\}$ and a constant $\lambda \in \mathbb{R}$. We find it remarkable that, although Dirac's original paper [3] about his monopole only appeared in 1931, Henri Poincaré investigated the exact same system of equations in a 1896 paper [25]. His analysis was a successful attempt to explain an experiment of the physicist Kristian Birkeland, which consisted of approaching one pole of a strong magnet near cathode rays, the other pole being far enough to be considered negligible. We thus call this one-body dynamical system the "Poincaré problem in $\dot{\mathbb{R}}^{3}$ ".

In this paper, we are interested in the generalization of this problem to $\mathrm{SU}(2)$ gauge theory. Recall that Dirac's monopole in $\mathbb{R}^{3}$ is obtained by radially extending the Hopf fibration $S^{3} \rightarrow S^{2}$ to a principal U(1)-bundle over $\dot{\mathbb{R}}^{3}[19,27,31]$. The same procedure using the next Hopf fibration $S^{7} \rightarrow S^{4}$ gives rise to non-Abelian analogue of the monopole in Euclidean space $\mathbb{R}^{5}$, known in the literature as Yang's monopole [20, 34]. It is a non-trivial SO(5)-symmetrical solution to the Yang-Mills equations in SU(2) gauge theory. Our main concern, which we call the "Poincaré problem in $\dot{\mathbb{R}}^{5}$ ", is for the classical motion of a charged particle in the presence of this monopole. The equations of motion are derived in Section 3 using a Kaluza-Klein formalism. In this context, the charge - which generalizes $\lambda$ in (1.1) - is a vector e rotating in $\mathbb{R}^{3}$.

The first system, (1.1), has been thoroughly studied in the literature $[5,6,7,8,10,12,14$, 23, 25, 26, 28]. The main result - as shown first by Poincaré - is that for every solution $\mathbf{r}$, there is a cone with vertex at the origin on which $\mathbf{r}$ is a geodesic (it follows from (1.1) that $|\dot{\mathbf{r}}|$ is constant). Moreover, Poincaré provided an explicit expression for the cone's direction and the angle at its vertex (which vary depending on the initial conditions and the charge $\lambda$ ). Since geodesics on cones are well understood, we get a complete description of the space of solutions. 
The main result of this paper is that this correspondence with geodesics on cones also holds for Yang's monopole, with suitable modifications. Given any solution $\mathbf{r}: I \rightarrow \dot{\mathbb{R}}^{5}$ of the equations of motion, there is a 4-dimensional cone with vertex at the origin of $\mathbb{R}^{5}$ on which $\mathbf{r}$ is a geodesic.

Our proof proceeds in two main steps. The first is the derivation of an explicit expression (given in Theorem 7.1) for the direction $\mathbf{L} \in \dot{\mathbb{R}}^{5}$ of the 4-dimensional cone on which the particle is a geodesic. The second is a general result (Theorem 6.1) about geodesics on higher dimensional cones that we prove here. This theorem states that for all $n \geq 2$, a geodesic on an $n$-dimensional cone $C$ is also a geodesic on a 2-dimensional cone embedded in $C$ with the same angle at the vertex, and conversely.

Moreover, this last result shows that particles in Yang's monopole follow geodesics on 2dimensional cones, and hence all solutions can be obtained explicitly, as was the case for Dirac's monopole.

There is a closely related problem called the "MICZ-Kepler system" [15, 35], which comes from generalizing the Kepler problem (for the motion of a particle under a central inversesquared attractive force in $\dot{\mathbb{R}}^{3}$ ) by adding a Lorentz force due to Dirac's monopole at the origin. It has also been generalized in $\dot{\mathbb{R}}^{5}$ using Yang's monopole [11], and in all Euclidean spaces $\dot{\mathbb{R}}^{n}$ by a construction due to Meng [16, 17, 18]. It was shown [1] that for all odd dimensions, the solutions to these systems are all conics. Moreover, Montgomery showed [22] that in any dimension, this system is equivalent to the classical Kepler problem on a cone (with no magnetic charge). It is thus natural to expect that the magnetic monopole alone would yield straight lines on cones (geodesics). Our paper shows that this is the case, at least for Dirac's and Yang's monopole.

The paper is organized as follows. In Section 2, we recall the classical treatment of the Poincaré problem in $\dot{\mathbb{R}}^{3}$.

In Section 3 we briefly review the Kaluza-Klein formalism for the motion of a charged particle in a Yang-Mills field $[2,9,13,24]$. For a principal $G$-bundle $P \rightarrow M$ with connection, the KaluzaKlein approach is to construct a particular $G$-invariant metric on $P$ from a metric on $M$ and an Ad-invariant metric on $\mathfrak{g}$. Then, projection on $M$ of the geodesics on $P$ defines motion of charged particles in $M$. The analogue of the charge is a vector rotating in $\mathfrak{g}$. The goal of this section is to provide coordinate expressions for the equations of motion. We note (see Montgomery [21]) that this formulation is equivalent to the ones used by Sternberg [30], Weinstein [32], and Wong [33].

In Section 4 we describe the extended Hopf bundles endowed with connections that give the Poincaré problem in $\dot{\mathbb{R}}^{3}$ and $\dot{\mathbb{R}}^{5}$. They are obtained by radially extending the Hopf fibrations $S^{2 n-1} \rightarrow S^{n}$ for $n=2,4$ to fibrations $\dot{\mathbb{R}}^{2 n} \rightarrow \dot{\mathbb{R}}^{n+1}$, and taking the connections corresponding to a horizontal subspace that is orthogonal to the vertical subspace in Euclidean space $\dot{\mathbb{R}}^{2 n}$. As a first example we apply the Kaluza-Klein formalism to $\dot{\mathbb{R}}^{4} \rightarrow \dot{\mathbb{R}}^{3}$ and show that we recover the equations of motion (1.1).

In Section 5 we derive the equations of motion of the Poincaré problem in $\dot{\mathbb{R}}^{5}$. That is, the one-body dynamical system for the motion of a charged particle in the field of Yang's monopole.

Section 6 is devoted to the study of geodesics on higher dimensional cones. This section is independent from the rest of the paper, but its conclusions will be crucial to the solution of the Poincaré problem in $\dot{\mathbb{R}}^{5}$.

Finally, in Section 7 we show that a charged particle in Yang's monopole must follow a geodesic on a 4-dimensional cone centred at the origin of $\mathbb{R}^{5}$. We give an explicit expression for the cone, and thus obtain a complete description of the space of solutions.

As a side remark, we note that there is a converse to the result of this paper. We prove here one implication, namely, that if $\mathbf{r}$ a solution to the Poincaré problem (in $\dot{\mathbb{R}}^{3}$ or $\dot{\mathbb{R}}^{5}$ ) then $\mathbf{r}$ is a geodesic on a cone with vertex at the origin. But we also have that for any cone centred at the origin (of $\mathbb{R}^{3}$ or $\mathbb{R}^{5}$ ) and any geodesic $\mathbf{r}$ on it, there is a unique charge ( $\lambda$ or $\mathbf{e}$ ) for which $\mathbf{r}$ is a solution to the Poincaré problem (in $\dot{\mathbb{R}}^{3}$ or $\dot{\mathbb{R}}^{5}$ ). For brevity we will not discuss this, but it can be proved with the theory presented in this paper. 


\section{Particle motion in Dirac's monopole}

Let us recall Poincaré's work [25] on the motion of a charged particle in the field of a single magnetic pole. Taking the pole to be centred at the origin, we find an electromagnetic field of the form

$$
\mathbf{E}=0, \quad \mathbf{B}=g \frac{\mathbf{r}}{r^{3}},
$$

for some constant $g \in \mathbb{R}, \mathbf{r} \in \dot{\mathbb{R}}^{3}:=\mathbb{R}^{3} \backslash\{0\}$ and $r=|\mathbf{r}|$. Assuming the particle is subject to the Lorentz force $\mathbf{F}=q(\mathbf{E}+\dot{\mathbf{r}} \times \mathbf{B})$, we get the equation of motion

$$
\ddot{\mathbf{r}}=\lambda \frac{\mathbf{r} \times \dot{\mathbf{r}}}{r^{3}}
$$

for some constant $\lambda \in \mathbb{R}$ depending on the strength $g$ of the magnet and the mass $m$ and charge $q$ of the particle. This is the system of ordinary differential equations that Poincaré analysed, and is also the one describing motion of a charged particle in the field of Dirac's monopole. Now, as Poincaré noticed, differentiation shows that the vector

$$
\mathbf{L}:=\mathbf{r} \times \dot{\mathbf{r}}+\lambda \frac{\mathbf{r}}{r}
$$

is constant. Taking the norm, we see that $\mathbf{L}=0$ if and only if $\lambda=0$ and $\dot{\mathbf{r}}$ is everywhere parallel to $\mathbf{r}$. This corresponds to motion at constant speed on a straight line that passes through the origin. Since those curves will come out often here and in subsequent sections, we give them the following name (a term borrowed from [1]).

Definition 2.1. A colliding curve is a curve $\mathbf{r}: I \rightarrow \dot{\mathbb{R}}^{n}$ such that $\dot{\mathbf{r}}$ is everywhere parallel to $\mathbf{r}$.

Now, suppose $\mathbf{r}$ is non-colliding. Then, the cosine of the angle between $\mathbf{r}$ and $\mathbf{L}$ is

$$
\cos \psi=\frac{\mathbf{r} \cdot \mathbf{L}}{|\mathbf{r}||\mathbf{L}|}=\frac{\lambda}{|\mathbf{L}|}
$$

which is constant. Hence, the particle moves on a cone directed along L. Furthermore, (2.1) shows that the acceleration is always normal to the surface of the cone, and so the particle follows a geodesic of that cone.

With this information in hand, the problem reduces to the geodesic equations on a cone in $\mathbb{R}^{3}$ - a standard problem. Note that the system (2.1) is invariant under rotation, so we may assume the cone is directed along the positive $z$-axis. Taking $t=0$ to be the point of closest approach to the origin, we find

$$
\mathbf{r}(t)=\sqrt{r_{0}^{2}+v_{0}^{2} t^{2}}\left(\sin \psi \cos \left(\frac{\arctan \left(v_{0} t / r_{0}\right)}{\sin \psi}\right), \sin \psi \sin \left(\frac{\arctan \left(v_{0} t / r_{0}\right)}{\sin \psi}\right), \cos \psi\right),
$$

where $r_{0}, v_{0}$ are the initial radius and velocity and $\psi$ is half the angle at the vertex of the cone. Moreover, equation (2.2) gives an explicit expression for the angle, namely, $\psi=\arctan \left(r_{0} v_{0} / \lambda\right)$. Fig. 1 shows a geodesic on a cone.

In this section, the equations of motion were derived classically by considering the "Coulomblike" magnetic field $\mathbf{B}=g \mathbf{r} /|\mathbf{r}|^{3}$ and the Lorentz force. But Dirac's monopole is also naturally described in terms of a connection on the radial extension of the Hopf bundle $S^{1} \rightarrow S^{3} \rightarrow S^{2}$. In Section 4 we will show that this approach together with the Kaluza-Klein formalism give rise to the exact same equations of motion. Motion in Yang's monopole in $\dot{\mathbb{R}}^{5}$ will be obtained this way but by using the next Hopf fibration $S^{3} \rightarrow S^{7} \rightarrow S^{4}$. 


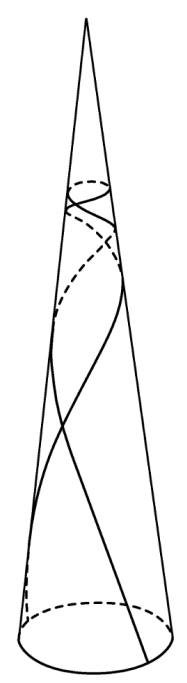

Figure 1. A geodesic on a cone.

\section{The Kaluza-Klein formalism}

Since Dirac's and Yang's monopole are more generally Yang-Mills fields, we need a way of obtaining the equations of motion of a particle in a general Yang-Mills field. There are several equivalent ways [21] of doing this, including the formulations of Sternberg [30], Weinstein [32], Wong [33], and Kerner [13]. In this paper we use the latter approach, which is known in the literature as the "Kaluza-Klein formalism". The goal of this section is to briefly review this formalism and to give coordinate expressions for the equations of motion. We closely follow the presentation of [9]. See also [2, 13, 24].

Let $\pi: P \rightarrow M$ be a principal bundle with structure group $G$ acting on $P$ to the right. We then have local sections $\sigma_{i}: U_{i} \rightarrow \pi^{-1}\left(U_{i}\right)$ such that the local trivialisations $\phi_{i}(p)=(\pi(p), a) \in U_{i} \times G$ correspond to the right action $p=\sigma(\pi(p)) \cdot a$. Let $\theta$ be a connection one-form on $P$. For an Ad-invariant metric $\langle$,$\rangle on the Lie algebra \mathfrak{g}$ of $G$ and a metric $g$ on $M$, define the following $G$-invariant metric on $P$,

$$
\left.\gamma(X, Y)\right|_{p}=\left.g\left(\pi_{*}(X), \pi_{*}(Y)\right)\right|_{\pi(p)}+\left\langle\left.\theta(X)\right|_{p},\left.\theta(Y)\right|_{p}\right\rangle .
$$

Then, $P$ with this metric $\gamma$ is a Riemmanian manifold whose geodesics projected to $M$ define the motion of charged particles in $M$, where the charge is a vector of constant magnitude in $\mathfrak{g}$.

We now set up the equations of motion in terms of a local coordinate system $\left\{x_{i}\right\}$ on an open neighbourhood $U \subseteq M$ and a basis $\left\{T_{k}\right\}$ for $\mathfrak{g}$. Let $T_{p} P=V_{p} \oplus H_{p}$ be the decomposition of the tangent space into a vertical and horizontal subspace. The action of $G$ induces a canonical isomorphism between $V_{p}$ and $\mathfrak{g}$, which gives fundamental vector fields $\left\{L_{k}\right\}$ on $P$ corresponding to $\left\{T_{k}\right\}$. Then, for a curve $t \mapsto p(t)$ in $P$, the tangent vector at $t$ is $\dot{p}(t)=v(t)+h(t)$, for some $v(t) \in V_{p(t)}$ and $h(t) \in H_{p(t)}$, and we may expand $v=v^{k} L_{k}$. The geodesic equations for the curve $p$ with respect to the metric $\gamma$ then become

$$
\begin{aligned}
& \ddot{x}^{\mu}+\Gamma_{\lambda \rho}^{\mu} \dot{x}^{\lambda} \dot{x}^{\rho}=\left\langle v, \tilde{F}_{\lambda \nu} \dot{x}^{\nu}\right\rangle g^{\lambda \mu}, \\
& \dot{v}^{k}=0
\end{aligned}
$$

where $\Gamma_{\lambda \rho}^{\mu}$ are the Christoffel symbols of the metric $g$ on $M$, and

$$
\tilde{F}=d \theta+\frac{1}{2}[\theta, \theta]=\frac{1}{2} \tilde{F}_{\mu \nu} \mathrm{d} x^{\mu} \wedge \mathrm{d} x^{\nu}
$$


is the curvature two-form. Let $\sigma: U \rightarrow \pi^{-1}(U)$ be the canonical local section and define the $\mathfrak{g}$-valued one-form

$$
A=A_{\mu} \mathrm{d} x^{\mu}=A_{\mu}^{k} T_{k} \mathrm{~d} x^{\mu}=\sigma^{*}(\theta),
$$

called the local gauge potential over $U$. Let $a \in G$ be the local trivialization $p \sim(\pi(p), a) \in$ $U \times G$, and let

$$
e=e^{k} T_{k}=a v a^{-1} .
$$

Finally, set $b_{i j}:=\left\langle T_{i}, T_{j}\right\rangle$, let $C_{i j}^{k}$ be the structure constants of $\mathfrak{g}$, and let

$$
F_{\mu \nu}=F_{\mu \nu}^{k} T_{k}=\partial_{\mu} A_{\nu}-\partial_{\nu} A_{\mu}+\left[A_{\mu}, A_{\nu}\right]
$$

be the curvature two-form in the $\sigma$-gauge. So $F_{\mu \nu}$ and $\tilde{F}_{\mu \nu}$ are related by $\tilde{F}_{\mu \nu}=a^{-1} F_{\mu \nu} a$. Then, the geodesic equations (3.1) and (3.2) are

$$
\begin{aligned}
& \ddot{x}^{\mu}+\Gamma_{\lambda \rho}^{\mu} \dot{x}^{\lambda} \dot{x}^{\rho}=b_{i j} e^{i} F_{\lambda \nu}^{j} \dot{x}^{\nu} g^{\lambda \mu}, \\
& \dot{e}^{k}+C_{i j}^{k} A_{\mu}^{i} e^{j} \dot{x}^{\mu}=0 .
\end{aligned}
$$

This system of ordinary differential equations defines the motion of a charged particle in $M$. The term on the right-hand side of (3.3) is the generalization of the Lorentz force. The vector $e=e^{k} T_{k} \in \mathfrak{g}$ is the analogue of the charge divided by the mass of the particle. Note that $e$ has magnitude $\langle e, e\rangle^{1 / 2}=\langle v, v\rangle^{1 / 2}$, which is constant by (3.2). However, unless $G$ is Abelian, $e$ itself is not in general constant.

\section{The extended Hopf bundles}

The purpose of this section is to define the principal bundles endowed with connections that describe Dirac's and Yang's monopole. The bundles are obtained by radially extending the Hopf bundles $S^{n-1} \rightarrow S^{2 n-1} \rightarrow S^{n}$, for $n=2,4$. We will first give a more abstract definition by means of the canonical projection of certain quotient spaces of the vector spaces $\dot{\mathbb{C}}^{2}$ and $\dot{\mathbb{H}}^{2}$. It will then lead to the desired bundles $\dot{\mathbb{R}}^{2 n} \rightarrow \dot{\mathbb{R}}^{n+1}$ by diffeomorphisms. Similar constructions can be found in [31] and [4].

Let $K$ be $\mathbb{C}$ or $\mathbb{H}$, and let $\dot{K}^{2}:=K^{2} \backslash\{(0,0)\}$. Let $\sim$ be the equivalence relation on $\dot{K}^{2}$ defined by $\left(z_{1}, z_{2}\right) \sim\left(w_{1}, w_{2}\right)$ if there is a unit norm $\lambda \in K$ such that $\left(z_{1}, z_{2}\right)=\left(w_{1} \lambda, w_{2} \lambda\right)$. The quotient of $\dot{K}^{2}$ by this relation gives an $(n+1)$-dimensional differentiable manifold $M$, and we define the extended Hopf map by the canonical projection

$$
\pi: \dot{K}^{2} \rightarrow M
$$

We get the structure of a principal bundle as follows. Let $S_{K}^{1}$ be the set of unit norm elements in $K$. We have $S_{\mathbb{C}}^{1}=\mathrm{U}(1)$ and $S_{\mathbb{H}}^{1}=\mathrm{SU}(2)$, so $S_{K}^{1}$ is a Lie group. It acts freely on $\dot{K}^{2}$ by $\left(z_{1}, z_{2}\right) \cdot \lambda=\left(z_{1} \lambda, z_{2} \lambda\right)$, and $M$ is the quotient space of this action. Moreover, $M$ is covered by the two open neighbourhoods

$$
U_{i}:=\left\{\left[\left(z_{1}, z_{2}\right)\right] \in M: z_{i} \neq 0\right\}, \quad i=1,2,
$$

over which we have the local trivializations

$$
\pi^{-1}\left(U_{i}\right) \rightarrow M \times S_{K}^{1}, \quad\left(z_{1}, z_{2}\right) \mapsto\left(\left[\left(z_{1}, z_{1}\right)\right], z_{i} /\left|z_{i}\right|\right), \quad i=1,2 .
$$


Thus, the extended Hopf map (4.1) is a principal $S_{K}^{1}$-bundle. Now, the principal bundle $S_{K}^{1} \rightarrow$ $\dot{\mathbb{R}}^{2 n} \rightarrow \dot{\mathbb{R}}^{n+1}$ is obtained from (4.1) by the identification of $K$ with $\mathbb{R}^{n}$ using the basis $\{1, i\}$ for $\mathbb{C}$ and $\{1, i, j, k\}$ for $\mathbb{H}$, and by the diffeomorphism

$$
f: M \rightarrow \dot{\mathbb{R}}^{n+1}, \quad\left[\left(z_{1}, z_{2}\right)\right] \mapsto\left(\frac{2 z_{1} z_{2}^{*}}{\sqrt{\left|z_{1}\right|^{2}+\left|z_{2}\right|^{2}}}, \frac{\left|z_{1}\right|^{2}-\left|z_{2}\right|^{2}}{\sqrt{\left|z_{1}\right|^{2}+\left|z_{2}\right|^{2}}}\right)
$$

where ${ }^{*}$ denotes conjugation in $K$. This construction gives the two principal bundles

$$
\mathrm{U}(1) \rightarrow \dot{\mathbb{R}}^{4} \rightarrow \dot{\mathbb{R}}^{3} \quad \text { and } \quad \mathrm{SU}(2) \rightarrow \dot{\mathbb{R}}^{8} \rightarrow \dot{\mathbb{R}}^{5}
$$

Motion will take place in Euclidean spaces $\dot{\mathbb{R}}^{3}$ and $\dot{\mathbb{R}}^{5}$.

Let us introduce the following set of coordinates on $M \cong \dot{\mathbb{R}}^{n+1}$.

$$
\begin{array}{lll}
\phi_{1}: U_{1} \subseteq M \rightarrow \mathbb{R}^{n} \times \mathbb{R}^{+}, & {\left[\left(z_{1}, z_{2}\right)\right]} & \mapsto\left(z_{2} z_{1}^{-1}, \sqrt{\left|z_{1}\right|^{2}+\left|z_{2}\right|^{2}}\right), \\
\phi_{2}: U_{2} \subseteq M \rightarrow \mathbb{R}^{n} \times \mathbb{R}^{+}, & {\left[\left(z_{1}, z_{2}\right)\right] \mapsto\left(z_{1} z_{2}^{-1}, \sqrt{\left|z_{1}\right|^{2}+\left|z_{2}\right|^{2}}\right) .}
\end{array}
$$

These coordinates are denoted $(\mathbf{u}, r)=\left(u_{1}, \ldots, u_{n}, r\right)$ and are related to the Cartesian coordinates $\left(x_{1}, \ldots, x_{n+1}\right)$ of $\dot{\mathbb{R}}^{n+1}$ by

$$
\begin{aligned}
& \left(x_{1}, \ldots, x_{n+1}\right)=\left(f \circ \phi_{1}^{-1}\right)(\mathbf{u}, r)=\left(\frac{2 r \mathbf{u}^{*}}{1+|\mathbf{u}|^{2}}, r \frac{1-|\mathbf{u}|^{2}}{1+|\mathbf{u}|^{2}}\right), \\
& \left(x_{1}, \ldots, x_{n+1}\right)=\left(f \circ \phi_{2}^{-1}\right)(\mathbf{u}, r)=\left(\frac{2 r \mathbf{u}}{|\mathbf{u}|^{2}+1}, r \frac{|\mathbf{u}|^{2}-1}{|\mathbf{u}|^{2}+1}\right) .
\end{aligned}
$$

An observation that will be crucial later is that $(\mathbf{u}, r)$ are precisely the stereographic projection coordinates from the south and north poles respectively. That is, for $\mathbf{r} \in \dot{\mathbb{R}}^{n+1}$, first project on the unit sphere by $\mathbf{r} \mapsto \mathbf{r} /|\mathbf{r}|$. Then, the stereographic projection of $\mathbf{r} /|\mathbf{r}|$ gives $\mathbf{u}=\left(u_{1}, \ldots, u_{n}\right)$, and the remaining coordinate $r$ is the magnitude of $\mathbf{r}$.

To apply the Kaluza-Klein formalism, we further need a connection on the bundle, a metric on $M \cong \dot{\mathbb{R}}^{n+1}$ and an Ad-invariant metric on $\mathfrak{g}$. The connection that gives the Poincaré problem is obtained by choosing a horizontal subspace that is orthogonal to the vertical subspace in Euclidean space $\dot{\mathbb{R}}^{2 n}$. The metric on $M$ is the one corresponding to the Euclidean metric on $\dot{\mathbb{R}}^{n+1}$. Since we observed that the coordinates $\left(u_{1}, \ldots, u_{n}, r\right)$ on $M$ are the stereographic projection coordinates of $\dot{\mathbb{R}}^{n+1}$, we know as a standard result that the metric on $M$ is

$$
g=\frac{4 r^{2} \sum_{i=1}^{n} \mathrm{~d} u_{i} \otimes \mathrm{d} u_{i}}{\left(1+u_{1}^{2}+\cdots+u_{n}^{2}\right)^{2}}+\mathrm{d} r \otimes \mathrm{d} r
$$

For the Ad-invariant metric on $\mathfrak{g}$ we take $\left\langle T_{i}, T_{j}\right\rangle:=\delta_{i j}$, where $\left\{T_{i}\right\}$ is a basis for $\mathfrak{g}$. When $G=\mathrm{U}(1)$, this basis is the imaginary number $T_{1}=i$, and when $G=\mathrm{SU}(2)$, it is $\left\{T_{1}=i\right.$, $\left.T_{2}=j, T_{3}=k\right\}$. It is straightforward to verify that $\left\langle T_{i}, T_{j}\right\rangle:=\delta_{i j}$ is Ad-invariant.

As an example, we apply the Kaluza-Klein formalism to the principal bundle $\mathrm{U}(1) \rightarrow \dot{\mathbb{R}}^{4} \rightarrow$ $\dot{\mathbb{R}}^{3}$ and show that we recover the equations of motion obtained in Section 2, i.e. those describing the classical motion of a charged particle in the field of Dirac's monopole.

The vertical subspace $V_{p} \subseteq T_{p} \dot{\mathbb{C}}^{2}$ for $p=\left(z_{1}, z_{2}\right) \in \dot{\mathbb{C}}^{2}$ is spanned by $\left.\frac{d}{d t}\right|_{t=0}\left(z_{1}, z_{2}\right) \cdot \exp (t i)=$ $\left(z_{1} i, z_{2} i\right)$. In the Cartesian coordinates $\left(x_{1}, x_{2}, x_{3}, x_{4}\right)=\left(z_{1}, z_{2}\right)$ of $\dot{\mathbb{R}}^{4}$, we have

$$
V_{p}=\operatorname{span}\left\{-x_{2} \frac{\partial}{\partial x_{1}}+x_{1} \frac{\partial}{\partial x_{2}}-x_{4} \frac{\partial}{\partial x_{3}}+x_{3} \frac{\partial}{\partial x_{4}}\right\} .
$$


We take the horizontal subspace $H_{p}$ to be the orthogonal complement of $V_{p}$. The corresponding connection one-form is then

$$
\theta=i \frac{-x_{2} \mathrm{~d} x_{1}+x_{1} \mathrm{~d} x_{2}-x_{4} \mathrm{~d} x_{3}+x_{3} \mathrm{~d} x_{4}}{x_{1}^{2}+\cdots+x_{4}^{3}} .
$$

This gives the local gauge potential over $U_{1}$

$$
A=\sigma_{1}^{*}(\theta)=i \frac{y d x-x d y}{2 r(z+r)}
$$

where $(x, y, z)$ are the Cartesian coordinates in $\dot{\mathbb{R}}^{3}$ and $r=\sqrt{x^{2}+y^{2}+z^{2}}$. The curvature then reads

$$
F=i \frac{-z d x \wedge d y+y d x \wedge d z-x d y \wedge d z}{2 r^{3}} .
$$

Inserting in the equations of motion (3.3) and (3.4), we get

$$
\ddot{x}=\frac{e}{2} \cdot \frac{y \dot{z}-z \dot{y}}{r^{3}}, \quad \ddot{y}=\frac{e}{2} \cdot \frac{z \dot{x}-x \dot{z}}{r^{3}}, \quad \ddot{z}=\frac{e}{2} \cdot \frac{x \dot{y}-y \dot{x}}{r^{3}}, \quad \dot{e}=0,
$$

which are precisely Poincaré's original equations (2.1) with $\lambda=e / 2$.

\section{The equations of motion of a particle in Yang's monopole}

In this section, we obtain the equations of motion of a charged particle in the presence of Yang's monopole in $\dot{\mathbb{R}}^{5}$ by applying the Kaluza-Klein formalism to the extended Hopf bundle $\mathrm{SU}(2) \rightarrow \dot{\mathbb{R}}^{8} \rightarrow \dot{\mathbb{R}}^{5}$ constructed in Section 4 .

To compute the vertical subspace, we use the right action of $\mathrm{SU}(2)$ to pullback the basis $\{i, j, k\}$ of $\mathfrak{s u}(2)$ to a basis for $V_{p}$. The basis vectors are $\left.L_{i}\right|_{p}:=\left.\frac{d}{d t}\right|_{t=0} p \cdot \exp \left(t T_{i}\right)$, and in the Cartesian coordinates $\left(x_{1}, \ldots, x_{8}\right)=\left(z_{1}, z_{2}\right)$ of $\dot{\mathbb{R}}^{8} \cong \dot{\mathbb{H}}^{2}$, we have

$$
\begin{aligned}
& L_{1}=\left(-x_{2}, x_{1}, x_{4},-x_{3},-x_{6}, x_{5}, x_{8},-x_{7}\right), \\
& L_{2}=\left(-x_{3},-x_{4}, x_{1}, x_{2},-x_{7},-x_{8}, x_{5}, x_{6}\right), \\
& L_{3}=\left(-x_{4}, x_{3},-x_{2}, x_{1},-x_{8}, x_{7},-x_{6}, x_{5}\right) .
\end{aligned}
$$

The connection one-form corresponding to a horizontal subspace that is orthogonal to $V_{p}$ is then

$$
\theta=\frac{1}{x_{1}^{2}+\cdots+x_{8}^{2}}\left(\begin{array}{cccccccc}
-x_{2} & x_{1} & x_{4} & -x_{3} & -x_{6} & x_{5} & x_{8} & -x_{7} \\
-x_{3} & -x_{4} & x_{1} & x_{2} & -x_{7} & -x_{8} & x_{5} & x_{6} \\
-x_{4} & x_{3} & -x_{2} & x_{1} & -x_{8} & x_{7} & -x_{6} & x_{5}
\end{array}\right)\left(\begin{array}{c}
\mathrm{d} x_{1} \\
\vdots \\
\mathrm{d} x_{8}
\end{array}\right)
$$

as expressed in the basis $\{i, j, k\}$ for $\mathfrak{s u}(2)$. We will now use the coordinate system $(\mathbf{u}, r)=$ $\left(u_{1}, \ldots, u_{4}, r\right)$ on $U_{2} \subseteq M \cong \mathbb{R}^{5}$ defined by (4.4). Recall that these are the stereographic projection coordinates from the north pole. Hence, we are working in $\dot{\mathbb{R}}^{5}$ minus the positive $x_{5^{-}}$ axis. Let $\sigma_{i}$ for $i=1,2$ be the canonical local sections induced by the local trivializations (4.2). On $U_{2}$ we obtain the gauge potential

$$
A=\sigma_{2}^{*}(\theta)=\frac{\mathbf{u}^{*} \mathrm{~d} \mathbf{u}-\mathrm{d} \mathbf{u}^{*} \mathbf{u}}{2\left(|\mathbf{u}|^{2}+1\right)},
$$

and the corresponding curvature

$$
F=\frac{\mathrm{d} \mathbf{u}^{*} \wedge \mathrm{d} \mathbf{u}}{2\left(|\mathbf{u}|^{2}+1\right)^{2}} .
$$


In matrix notation, we have $A=\mathbf{A d} \mathbf{u}$, where

$$
\mathbf{A}:=\frac{1}{|\mathbf{u}|^{2}+1}\left(\begin{array}{cccc}
-u_{2} & u_{1} & u_{4} & -u_{3} \\
-u_{3} & -u_{4} & u_{1} & u_{2} \\
-u_{4} & u_{3} & -u_{2} & u_{1}
\end{array}\right)=:\left(\begin{array}{c}
\mathbf{A}_{1} \\
\mathbf{A}_{2} \\
\mathbf{A}_{3}
\end{array}\right) \text {. }
$$

Inserting in the equations of motions (3.3) and (3.4) of the Kaluza-Klein formalism, we get the system of differential equations

$$
\begin{aligned}
& \ddot{\mathbf{u}}+\frac{2|\dot{\mathbf{u}}|^{2} \mathbf{u}-4(\mathbf{u} \cdot \dot{\mathbf{u}}) \dot{\mathbf{u}}}{|\mathbf{u}|^{2}+1}+\frac{2 \dot{r} \dot{\mathbf{u}}}{r}=\frac{\mathbf{E} \dot{\mathbf{u}}}{2 r^{2}}, \\
& \ddot{r}-\frac{4 r|\dot{\mathbf{u}}|^{2}}{\left(|\mathbf{u}|^{2}+1\right)^{2}}=0, \\
& \dot{\mathbf{e}}+2 \mathbf{B e}=0
\end{aligned}
$$

where $\mathbf{e}=\left(e^{1}, e^{2}, e^{3}\right)$ and

$$
\mathbf{E}:=\left(\begin{array}{cccc}
0 & e^{1} & e^{2} & e^{3} \\
-e^{1} & 0 & -e^{3} & e^{2} \\
-e^{2} & e^{3} & 0 & -e^{1} \\
-e^{3} & -e^{2} & e^{1} & 0,
\end{array}\right), \quad \mathbf{B}:=\left(\begin{array}{ccc}
0 & -B_{3} & B_{2} \\
B_{3} & 0 & -B_{1} \\
-B_{2} & B_{1} & 0
\end{array}\right), \quad B_{i}:=\mathbf{A}_{i} \cdot \dot{\mathbf{u}} .
$$

These equations describe the motion of a charged particle in the field of Yang's monopole at the origin of Euclidean space $\mathbb{R}^{5}$. The vector $\mathbf{e}$ is interpreted as the charge of the particle, and $\left(\frac{1}{2 r^{2}} \mathbf{E} \dot{\mathbf{u}}, 0\right) \in \mathbb{R}^{4} \times \mathbb{R}^{+}$is the analogue of the Lorentz force. Note that (5.3) immediately gives $\mathbf{e} \cdot \dot{\mathbf{e}}=0$, and so $\mathbf{e}$ has constant magnitude, as anticipated in the general formulation of Section 3 .

\section{Some facts about cones and their geodesics}

The solutions to the equations of motion (5.1), (5.2) and (5.3) will be investigated in Section 7. Some crucial results that we will need can be stated as general facts about higher dimensional cones and their geodesics. Hence, we put them in this separate section, which is completely independent from the rest of the paper. The main goal is Theorem 6.1 and its two corollaries.

First of all, we need a clear definition of what we mean by a $k$-dimensional cone in $\mathbb{R}^{n}$, for $k<n$. In this paper all cones will have their vertex at the origin. Before the general definition, here is the most basic classical one.

Definition 6.1. The cone of aperture $\psi \in(0, \pi / 2]$ directed along $\mathbf{L} \in \dot{\mathbb{R}}^{n}$ is the set of all points $\mathbf{r} \in \dot{\mathbb{R}}^{n}$ satisfying

$$
\frac{\mathbf{r} \cdot \mathbf{L}}{|\mathbf{r}||\mathbf{L}|}=\cos \psi
$$

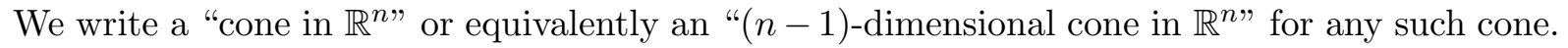

To generalize this definition to $k$-dimensional cones in $\mathbb{R}^{n}$ for any $k<n$, we need the following observation. In the definition of a cone, equation (6.1) can be rewritten $(\mathbf{r} /|\mathbf{r}|) \cdot \mathbf{L}=b$, where $b=|\mathbf{L}| \cos \psi$ is a constant. Thus, the cone is the set of all points in $\mathbb{R}^{n}$ such that when projected on the unit sphere $S^{n-1}$ they lie in the fixed affine hyperplane $\left\{\mathbf{x} \in \mathbb{R}^{n}: \mathbf{x} \cdot \mathbf{L}=b\right\}-$ see Fig. 2. This motivates the following definition.

Definition 6.2. Let $P$ be an affine $k$-dimensional plane in $\mathbb{R}^{n}$ that intersects with $S^{n-1}$ in more than one point. The cone generated by $P$ in $\mathbb{R}^{n}$ is the set of all points $\mathbf{r} \in \dot{\mathbb{R}}^{n}$ such that $\mathbf{r} /|\mathbf{r}| \in P$. We write a " $k$-dimensional cone in $\mathbb{R}^{n}$ " for any such set. 


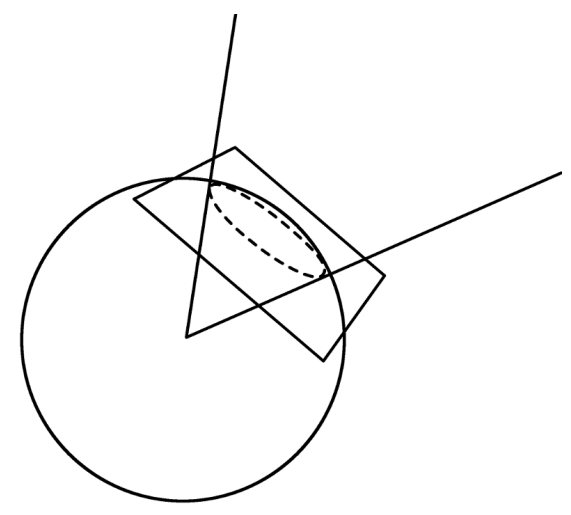

Figure 2. A cone is defined by radially extending the intersection of an affine plane with the unit sphere.

To better understand this definition, we first remark the following basic fact.

Proposition 6.1. Let $P$ be a $k$-dimensional affine plane in $\mathbb{R}^{n}$ intersecting the unit sphere $S^{n-1}$ in more than one point. Then, $P \cap S^{n-1}$ is a $(k-1)$-sphere.

This proposition shows that a $k$-dimensional cone in $\mathbb{R}^{n}$ is parametrized by a point in a $(k-1)$ sphere and a positive number $r>0$. For example, a 2-dimensional cone in $\mathbb{R}^{3}$ intersects the unit sphere on a circle, and so each point of the cone is uniquely defined by a point on this circle and a radius $r>0$. Here is the proof of the proposition.

Proof. Let $P=\mathbf{a}+U$, where $\mathbf{a} \in \mathbb{R}^{n}$ and $U$ is a $k$-dimensional subspace of $\mathbb{R}^{n}$. Without loss of generality, we may assume that $\mathbf{a}$ is orthogonal to $U$. Choose an orthonormal basis $\left\{\mathbf{v}_{1}, \ldots, \mathbf{v}_{k}\right\}$ for $U$. Then,

$$
\begin{aligned}
P \cap S^{n-1} & =\left\{\mathbf{a}+x_{1} \mathbf{v}_{1}+\cdots+x_{k} \mathbf{v}_{k}: x_{i} \in \mathbb{R},\left|\mathbf{a}+x_{1} \mathbf{v}_{1}+\cdots+x_{k} \mathbf{v}_{k}\right|^{2}=1\right\} \\
& =\left\{\mathbf{a}+x_{1} \mathbf{v}_{1}+\cdots+x_{k} \mathbf{v}_{k}: x_{i} \in \mathbb{R}, x_{1}^{2}+\cdots+x_{k}^{2}=1-|\mathbf{a}|^{2}\right\},
\end{aligned}
$$

which is a $(k-1)$-sphere of radius $\sqrt{1-|\mathbf{a}|^{2}}$ centred at $\mathbf{a}$ in $\mathbb{R}^{n}$.

The following proposition shows that a $k$-dimensional cone in $\mathbb{R}^{n}$, as of Definition 6.2 , is in a sense exactly the same as the classical Definition 6.1 of a cone in $\mathbb{R}^{k+1}$.

Proposition 6.2. Let $C$ be a k-dimensional cone in $\mathbb{R}^{n}$ for $n>k$. There is a cone $D$ in $\mathbb{R}^{k+1}$ directed along $(0, \ldots, 0,1) \in \mathbb{R}^{k+1}$, and a matrix $\mathbf{R} \in \mathrm{SO}(n)$ such that

$$
\mathbf{R}(C)=\left\{\left(x_{1}, \ldots, x_{k+1}, 0, \ldots, 0\right) \in \mathbb{R}^{n}:\left(x_{1}, \ldots, x_{k+1}\right) \in D\right\} .
$$

Proof. Let $P$ be the $k$-dimensional affine plane in $\mathbb{R}^{n}$ that generates $C$. It is straightforward to see that there exists $\mathbf{R} \in \mathrm{SO}(n)$ such that

$$
\mathbf{R}(P)=\left\{\left(x_{1}, \ldots, x_{k}, a, 0, \ldots, 0\right): x_{i} \in \mathbb{R}\right\},
$$

for some $a \geq 0$. Note that since $P$ intersects $S^{n-1}$ in more than one point, we have $0 \leq a<1$, and hence there is an angle $\psi \in(0, \pi / 2]$ such that $a=\cos \psi$. Letting $\mathbf{L}=(0, \ldots, 0,1) \in \mathbb{R}^{k+1}$, we get

$$
\begin{aligned}
\mathbf{R}(C) & =\left\{\mathbf{R}(\mathbf{r}) \in \mathbb{R}^{n}: \mathbf{r} /|\mathbf{r}| \in P\right\}=\left\{\mathbf{r} \in \mathbb{R}^{n}: \mathbf{r} /|\mathbf{r}| \in \mathbf{R}(P)\right\} \\
& =\left\{\mathbf{r} \in \mathbb{R}^{n}: \mathbf{r} /|\mathbf{r}|=\left(x_{1}, \ldots, x_{k}, \cos \psi, 0, \ldots, 0\right) \text { for some } x_{i} \in \mathbb{R}\right\}
\end{aligned}
$$




$$
\begin{aligned}
& =\left\{(\tilde{\mathbf{r}}, 0, \ldots, 0) \in \mathbb{R}^{k+1} \times \mathbb{R}^{n-k-1}: \frac{\tilde{\mathbf{r}} \cdot \mathbf{L}}{|\tilde{\mathbf{r}}||\mathbf{L}|}=\cos \psi\right\} \\
& =\left\{\left(x_{1}, \ldots, x_{k+1}, 0, \ldots, 0\right) \in \mathbb{R}^{n}:\left(x_{1}, \ldots, x_{k+1}\right) \in D\right\},
\end{aligned}
$$

where $D$ is the cone of aperture $\psi$ directed along $\mathbf{L}$ in $\dot{\mathbb{R}}^{k+1}$.

This proposition and its proof allow us to make the following definition.

Definition 6.3. Let $C$ be a $k$-dimensional cone in $\mathbb{R}^{n}$, and let $P$ be the affine $k$-dimensional plane generating $C$. Write $P=\mathbf{a}+U$ for $\mathbf{a} \in U^{\perp}$. The aperture of $C$ is the number $\psi \in(0, \pi / 2]$ for which $\cos \psi=|\mathbf{a}|$.

It is straightforward to verify that this is well-defined ( $\mathbf{a}$ is unique and $0 \leq|\mathbf{a}|<1$ ) and that it matches the classical Definition 6.1. For a 2-dimensional cone $C$ in $\mathbb{R}^{3}$, our definition of the aperture is half the angle at the vertex of $C$.

Consider a 1-dimensional cone of aperture $\psi$ in $\mathbb{R}^{3}$ (two non-parallel rays coming from the origin). It is intuitively clear that there is a unique 2 -dimensional cone of aperture $\psi$ containing it. Indeed, just rotate the two rays about the bisector, and it will give the desired cone. This principle of "unique embedding" is indeed true, and generalizes as follows.

Proposition 6.3. Let $D$ be a $k$-dimensional cone of aperture $\psi$ in $\mathbb{R}^{n}$, for any $k<n$. There is a unique $(n-1)$-dimensional cone of aperture $\psi$ containing $D$.

Proof. Let $Q=\mathbf{a}+U$ be the $k$-dimensional affine plane generating $D$, and assume $\mathbf{a} \in U^{\perp}$ so that $\cos \psi=|\mathbf{a}|$. Then, $U \subseteq(\operatorname{span}\{\mathbf{a}\})^{\perp}$, so the hyperplane $P=\mathbf{a}+(\operatorname{span}\{\mathbf{a}\})^{\perp}$ generates an $(n-$ 1)-dimensional cone of aperture $\psi$ containing $D$. This shows existence. For uniqueness, suppose that $\tilde{P}=\mathbf{b}+V, \mathbf{b} \in V^{\perp}$, generates an $(n-1)$-dimensional cone of aperture $\psi$ containing $D$. Write $\mathbf{b}=\mathbf{a}+\mathbf{c}$ for $\mathbf{c} \in(\operatorname{span}\{\mathbf{a}\})^{\perp}$. Then, $|\mathbf{a}|^{2}=\cos ^{2} \psi=|\mathbf{b}|^{2}=|\mathbf{a}|^{2}+|\mathbf{c}|^{2}$, so $|\mathbf{c}|^{2}=0$ and hence $\mathbf{b}=\mathbf{a}$. Then, $\mathbf{a} \in V^{\perp}$ so we have $V \subseteq(\operatorname{span}\{\mathbf{a}\})^{\perp}$. But $\operatorname{dim} V=n-1=\operatorname{dim}(\operatorname{span}\{\mathbf{a}\})^{\perp}$, so $V=(\operatorname{span}\{\mathbf{a}\})^{\perp}$, whence $\tilde{P}=P$.

We will now start to investigate geodesics on cones. First, let us give a clear definition.

Definition 6.4. Let $C$ be a $k$-dimensional cone in $\mathbb{R}^{n}$ together with the metric inherited from the ambient Euclidean space $\mathbb{R}^{n}$ and let $\frac{D}{d t}$ be the corresponding covariant derivative. We call a geodesic on $C$ a differentiable map $\mathbf{r}: I \rightarrow \dot{\mathbb{R}}^{n}$, from an open interval $I \subseteq \mathbb{R}$ to $\dot{\mathbb{R}}^{n}$, such that $\mathbf{r}(I) \subseteq C$ and $\frac{D}{d t} \dot{\mathbf{r}}(t)=0$ for all $t \in I$.

Note that we do not assume that a geodesic is parametrized by arclength, and hence can have any (constant) speed.

We will now need a parametrization for an arbitrary $n$-dimensional cone $C$ in $\mathbb{R}^{n+1}$. Since for any $\mathbf{R} \in \mathrm{SO}(n+1)$, a curve $\mathbf{r}$ is a geodesic on a cone $C$ if and only if $\mathbf{R}(\mathbf{r})$ is a geodesic on $\mathbf{R}(C)$, we may assume without loss of generality that $C$ is directed along $\mathbf{L}=(0, \ldots, 0,1) \in \mathbb{R}^{n+1}$. Let $\psi \in(0, \pi / 2]$ be the aperture of $C$. Then,

$$
\begin{aligned}
C & =\left\{\left(x_{1}, \ldots, x_{n+1}\right) \in \mathbb{R}^{n+1}: x_{n+1}=\cos \psi \sqrt{x_{1}^{2}+\cdots+x_{n+1}^{2}}\right\} \\
& =\left\{\left(x_{1}, \ldots, x_{n+1}\right) \in \mathbb{R}^{n+1}: x_{n+1}^{2} \sin ^{2} \psi=\left(x_{1}^{2}+\cdots+x_{n}^{2}\right) \cos ^{2} \psi \text { and } x_{n+1}>0\right\} \\
& =\left\{\left(x_{1} r \sin \psi, \ldots, x_{n} r \sin \psi, r \cos \psi\right):\left(x_{1}, \ldots, x_{n}\right) \in S^{n-1} \text { and } r>0\right\} .
\end{aligned}
$$

Therefore, any parametrization of the unit sphere $S^{n-1}$ will give a natural parametrization of the cone $C$. We choose the stereographic projection coordinates from the north pole:

$$
\left(x_{1}, \ldots, x_{n}\right)=\left(\frac{2 v_{1}}{\sum_{i} v_{i}^{2}+1}, \ldots, \frac{2 v_{n-1}}{\sum_{i} v_{i}^{2}+1}, \frac{\sum_{i} v_{i}^{2}-1}{\sum_{i} v_{i}^{2}+1}\right)=\left(\frac{2 \mathbf{v}}{|\mathbf{v}|^{2}+1}, \frac{|\mathbf{v}|^{2}-1}{|\mathbf{v}|^{2}+1}\right),
$$


where $\mathbf{v}=\left(v_{1}, \ldots, v_{n-1}\right)$. This defines a coordinate system $\phi: \mathbb{R}^{n-1} \times \mathbb{R}^{+} \rightarrow C$ for the cone $C$ by

$$
\phi(\mathbf{v}, r)=\left(\frac{2 \mathbf{v}}{|\mathbf{v}|^{2}+1} r \sin \psi, \frac{|\mathbf{v}|^{2}-1}{|\mathbf{v}|^{2}+1} r \sin \psi, r \cos \psi\right),
$$

with inverse

$$
\phi^{-1}\left(x_{1}, \ldots, x_{n+1}\right)=\left(\frac{x_{1}}{r \sin \psi-x_{n}}, \ldots, \frac{x_{n-1}}{r \sin \psi-x_{n}}, r\right),
$$

where $r:=\left(x_{1}^{2}+\cdots+x_{n+1}^{2}\right)^{1 / 2}$. Now, the metric $g$ on $C$ is the one inherited from the ambient Euclidean space $\mathbb{R}^{n+1}$. In this coordinate system we get

$$
g=\frac{4 r^{2} \sin ^{2} \psi}{\left(|\mathbf{v}|^{2}+1\right)^{2}} \sum_{i=1}^{n-1} \mathrm{~d} v_{i} \otimes \mathrm{d} v_{i}+\mathrm{d} r \otimes \mathrm{d} r .
$$

The Christoffel symbols corresponding to this metric are

$$
\Gamma_{i j}^{k}= \begin{cases}2 \frac{v_{k} \delta_{i j}-v_{i} \delta_{j k}-v_{j} \delta_{k i}}{|\mathbf{v}|^{2}+1}, & i \neq n, \quad j \neq n, \quad k \neq n, \\ -\frac{4 r \sin ^{2} \psi}{\left(|\mathbf{v}|^{2}+1\right)^{2}} \delta_{i j}+\frac{\delta_{i k}+\delta_{j k}}{r}, & \text { one and only one of } i, j \text { or } k \text { is equal to } n \\ 0, & \text { else. }\end{cases}
$$

The geodesic equations are then

$$
\begin{aligned}
& \ddot{\mathbf{v}}+\frac{2|\dot{\mathbf{v}}|^{2} \mathbf{v}-4(\mathbf{v} \cdot \dot{\mathbf{v}}) \dot{\mathbf{v}}}{|\mathbf{v}|^{2}+1}+\frac{2 \dot{r} \dot{\mathbf{v}}}{r}=0, \\
& \ddot{r}-\frac{4 r \sin ^{2} \psi|\dot{\mathbf{v}}|^{2}}{\left(|\mathbf{v}|^{2}+1\right)^{2}}=0 .
\end{aligned}
$$

The most important result of this section is the following.

Theorem 6.1. Let $\mathbf{r}: I \rightarrow \dot{\mathbb{R}}^{n+1}$ be a non-colliding curve, where $n \geq 2$. Then, $\mathbf{r}$ is a geodesic on an $n$-dimensional cone $C$ if and only if $\mathbf{r}$ is a geodesic on a 2-dimensional cone $D \subseteq C$ of the same aperture.

Proof. We first show that if $\mathbf{r}: I \rightarrow \dot{\mathbb{R}}^{n+1}$ is a non-colliding geodesic on $C$, then $\mathbf{r}(I)$ is in a 2-dimensional cone $D \subseteq C$. By definition, we have to show that the curve $\alpha: I \rightarrow \mathbb{R}^{n+1}$ defined by $\alpha(t):=\mathbf{r}(t) /|\mathbf{r}(t)|$ lies on a fixed 2-dimensional plane. To do that, it suffices to show that $\{\dot{\alpha}, \ddot{\alpha}\}$ is everywhere linearly independent while $\{\dot{\alpha}, \ddot{\alpha}, \dddot{\alpha}\}$ is everywhere linearly dependent (see [29, Chapter 7, Part B, Theorem 5]).

To show that $\{\dot{\alpha}, \ddot{\alpha}\}$ is everywhere linearly independent, suppose that at some point $t_{0} \in I$ we have $\ddot{\alpha}=\lambda \dot{\alpha}$ for some $\lambda \in \mathbb{R}$. Since $\alpha \cdot \alpha=1$, we get $\alpha \cdot \ddot{\alpha}=\lambda \alpha \cdot \dot{\alpha}=0$. Taking the second derivative on both sides of $\alpha \cdot \alpha=1$, we then find $\dot{\alpha} \cdot \dot{\alpha}=0$ at $t_{0}$, and so $\dot{\mathbf{r}}\left(t_{0}\right)$ is parallel to $\mathbf{r}\left(t_{0}\right)$. Hence, we can form a colliding curve $\tilde{\mathbf{r}}(t):=\mathbf{r}\left(t_{0}\right)+\left(t-t_{0}\right) \dot{\mathbf{r}}\left(t_{0}\right)$, with $\tilde{\mathbf{r}}\left(t_{0}\right)=\mathbf{r}\left(t_{0}\right)$ and $\dot{\tilde{\mathbf{r}}}\left(t_{0}\right)=\dot{\mathbf{r}}\left(t_{0}\right)$. But $\tilde{\mathbf{r}}$ is solution to the geodesic equations (6.4) and (6.5), so by uniqueness we have $\mathbf{r}=\tilde{\mathbf{r}}$. This contradicts the assumption that $\mathbf{r}$ is non-colliding.

Now, to show that $\{\dot{\alpha}, \ddot{\alpha}, \ddot{\alpha}\}$ is everywhere linearly dependent, we will show an explicit nontrivial linear dependence. In the parametrization $(\mathbf{v}, r)$ of the cone $(6.2)$, we have

$$
\alpha=\left(\frac{2 \mathbf{v}}{|\mathbf{v}|^{2}+1} \sin \psi, \frac{|\mathbf{v}|^{2}-1}{|\mathbf{v}|^{2}+1} \sin \psi, \cos \psi\right)
$$


To compute the derivatives $\dot{\alpha}, \ddot{\alpha}, \dddot{\alpha}$, we insert the geodesic equations (6.4) and (6.5) to eliminate all second derivatives of $(\mathbf{v}, r)$. We obtain

$$
\begin{aligned}
\dot{\alpha}= & \frac{2 \sin \psi}{\left(|\mathbf{v}|^{2}+1\right)^{2}}\left(\left(|\mathbf{v}|^{2}+1\right) \dot{\mathbf{v}}-2(\mathbf{v} \cdot \dot{\mathbf{v}}) \mathbf{v}, 2 \mathbf{v} \cdot \dot{\mathbf{v}}, 0\right), \\
\ddot{\alpha}= & \frac{8 \sin \psi}{\left(|\mathbf{v}|^{2}+1\right)^{3}}\left(-|\dot{\mathbf{v}}|^{2} \mathbf{v}+\frac{\dot{r}}{2 r}\left(|\mathbf{v}|^{2}+1\right)\left(2(\mathbf{v} \cdot \dot{\mathbf{v}}) \mathbf{v}-\left(|\mathbf{v}|^{2}+1\right) \dot{\mathbf{v}}\right),\right. \\
& \left.-\frac{1}{2}|\dot{\mathbf{v}}|^{2}\left(|\mathbf{v}|^{2}-1\right)-\frac{\dot{r}}{r}\left(|\mathbf{v}|^{2}+1\right) \mathbf{v} \cdot \dot{\mathbf{v}}, 0\right), \\
\dddot{\alpha}= & \frac{2 \sin \psi}{\left(|\mathbf{v}|^{2}+1\right)^{2}}\left(\frac{6 \dot{r}^{2}}{r^{2}}-\frac{4\left(1+2 \sin ^{2} \psi\right)|\dot{\mathbf{v}}|^{2}}{\left(|\mathbf{v}|^{2}+1\right)^{2}}\right)\left(\left(|\mathbf{v}|^{2}+1\right) \dot{\mathbf{v}}-2(\mathbf{v} \cdot \dot{\mathbf{v}}) \mathbf{v}, 2 \mathbf{v} \cdot \dot{\mathbf{v}}, 0\right) \\
& -\frac{48 \dot{r} \sin \psi}{r\left(|\mathbf{v}|^{2}+1\right)^{3}}\left(-|\dot{\mathbf{v}}|^{2} \mathbf{v},-\frac{1}{2}|\dot{\mathbf{v}}|^{2}\left(|\mathbf{v}|^{2}-1\right), 0\right) .
\end{aligned}
$$

We then find that these expressions satisfy the relation

$$
\left(\frac{4|\dot{\mathbf{v}}|^{2}\left(1+2 \sin ^{2} \psi\right)}{\left(|\mathbf{v}|^{2}+1\right)^{2}}+\frac{6 \dot{r}^{2}}{r^{2}}\right) \dot{\alpha}+\frac{6 \dot{r}}{r} \ddot{\alpha}+\dddot{\alpha}=0
$$

So $\alpha$ is contained in a 2-dimensional plane and hence $\mathbf{r}$ is contained in a 2-dimensional cone $D$. Moreover, $D \subseteq C$ for the following reason. Let $Q$ be the affine 2-dimensional plane generating $D$, and let $P$ be the affine hyperplane generating $C$. Since $\mathbf{r}$ is non-colliding, we can find three distinct points in $\alpha(I) \subseteq Q \cap S^{n}$. But $Q \cap S^{n}$ is a circle (Proposition 6.1), so we have three non-collinear points of $Q \cap P$. Since an affine 2-dimensional plane is uniquely specified by three non-collinear points, we have $Q \subseteq P$, whence $D \subseteq C$.

We will now show that $\mathbf{r}$ is a geodesic on $D$ and that $D$ has aperture $\psi$. Still assuming the parametrization (6.2) for $C$, we have

$$
P=\left\{\left(x_{1}, \ldots, x_{n}, \cos \psi\right): x_{i} \in \mathbb{R}\right\},
$$

whence

$$
Q=(0, \ldots, 0, \cos \psi)+\mathbf{a}+\operatorname{span}\left\{\mathbf{w}_{1}, \mathbf{w}_{2}\right\},
$$

for some $\mathbf{a}, \mathbf{w}_{1}, \mathbf{w}_{2} \in\left\{\left(x_{1}, \ldots, x_{n}, 0\right): x_{i} \in \mathbb{R}\right\}$. But note that in the parametrization (6.2), $C$ has an $\mathrm{SO}(n)$ symmetry in its first $n$ components. Hence, we may assume that

$$
Q=\left\{\left(x_{1}, x_{2}, 0, \ldots, 0, a, \cos \psi\right): x_{1}, x_{2} \in \mathbb{R}\right\}
$$

for some $a \geq 0$. Since $Q \cap S^{n}$ contains more than one point, we have $a^{2}<1-\cos ^{2} \psi=\sin ^{2} \psi$, or equivalently, $a=\cos \varphi \sin \psi$ for some $\varphi \in(0, \pi / 2]$. Therefore,

$$
\begin{aligned}
D & =\left\{r\left(x_{1}, x_{2}, 0, \ldots, 0, \cos \varphi \sin \psi, \cos \psi\right): r>0, x_{1}^{2}+x_{2}^{2}+\cos ^{2} \varphi \sin ^{2} \psi+\cos ^{2} \psi=1\right\} \\
& =\left\{r\left(x_{1}, x_{2}, 0, \ldots, 0, \cos \varphi \sin \psi, \cos \psi\right): r>0, x_{1}^{2}+x_{2}^{2}=\sin ^{2} \varphi \sin ^{2} \psi\right\} \\
& =\{(r \cos \theta \sin \varphi \sin \psi, r \sin \theta \sin \varphi \sin \psi, 0, \ldots, 0, r \cos \varphi \sin \psi, r \cos \psi): r>0, \theta \in \mathbb{R}\},
\end{aligned}
$$

and so

$$
\mathbf{r}(t)=(r(t) \cos \theta(t) \sin \varphi \sin \psi, r(t) \sin \theta(t) \sin \varphi \sin \psi, 0, \ldots, 0, r(t) \cos \varphi \sin \psi, r(t) \cos \psi)
$$

for some functions $\theta: I \rightarrow \mathbb{R}$ and $r: I \rightarrow \mathbb{R}^{+}$. Now, using the inverse transformation (6.3), we can express $\mathbf{r}$ in the coordinates $(\mathbf{v}, r)=\left(v_{1}, \ldots, v_{n-1}, r\right)$ parametrizing $C$. We find

$$
(\mathbf{v}, r)=\left(\cos \theta \cot \frac{\varphi}{2}, \sin \theta \cot \frac{\varphi}{2}, 0, \ldots, 0, r\right) .
$$


Assuming that $\mathbf{r}$ has this form, the geodesic equations (6.4) and (6.5) for $\mathbf{r}$ on $C$ are equivalent to

$$
\begin{aligned}
& \cos \varphi=0, \\
& \ddot{\theta}+2 \dot{r} \dot{\theta} / r=0, \\
& \ddot{r}-r \dot{\theta}^{2} \sin ^{2} \psi=0 .
\end{aligned}
$$

The first equation shows that $D$ is the 2 -dimensional cone of aperture $\psi$ given by

$$
D=\{(r \cos \theta \sin \psi, r \sin \theta \sin \psi, 0, \ldots, 0, r \cos \psi): r>0, \theta \in \mathbb{R}\}
$$

and the last two equations are precisely the geodesic equations for $\mathbf{r}$ on $D$.

Conversely, this also shows that any geodesic of $D$ is a geodesic of $C$. Hence, by the $\mathrm{SO}(n)$ symmetry of $C$ in its first $n$ components (in (6.2)) we get that any geodesic on a 2-dimensional cone of aperture $\psi$ embedded in $C$ is a geodesic on $C$.

The following two corollaries will be important for the next section, when we will investigate the motion of a charged particle in the field of Yang's monopole.

Corollary 6.1. Let $\mathbf{r}: I \rightarrow \dot{\mathbb{R}}^{n+1}$ be a non-colliding curve, where $n \geq 2$. If $\mathbf{r}$ lies on a 2dimensional cone $D$ of aperture $\psi$ and $\ddot{\mathbf{r}}$ is orthogonal to $\mathbf{r}$ and $\dot{\mathbf{r}}$, then $\mathbf{r}$ is a geodesic on $D$ and hence on the unique $n$-dimensional cone of aperture $\psi$ containing $D$.

Proof. By rotation symmetry, we may assume that the 2-dimensional cone $D$ on which $\mathbf{r}$ lies is of the form (6.6), so that

$$
\mathbf{r}(t)=(r(t) \cos \theta(t) \sin \psi, r(t) \sin \theta(t) \sin \psi, 0, \ldots, 0, r(t) \cos \psi),
$$

for some functions $r: I \rightarrow \mathbb{R}^{+}$and $\theta: I \rightarrow \mathbb{R}$. This defines a space curve $\mathbf{p}: I \rightarrow \mathbb{R}^{3}$ by taking the three non-zero components of $\mathbf{r}$. We have that $\mathbf{p}$ lies on the cone $\tilde{D} \subseteq \mathbb{R}^{3}$ of aperture $\psi$ directed along $(0,0,1)$, and $\ddot{\mathbf{p}}$ is orthogonal to $\mathbf{p}$ and $\dot{\mathbf{p}}$. Hence, $\ddot{\mathbf{p}}$ is always normal to the surface of the cone $\tilde{D}$, so $\mathbf{p}$ is a geodesic on $\tilde{D}$. Therefore, $\mathbf{r}$ is a geodesic on $D$. Now, Proposition 6.3 shows that there exists a unique $n$-dimensional cone $C$ of aperture $\psi$ containing $D$, and then Theorem 6.1 shows that $\mathbf{r}$ is a geodesic on that cone.

Corollary 6.2. Let $\mathbf{r}: I \rightarrow \dot{\mathbb{R}}^{n}$ be a non-colliding geodesic on a $k$-dimensional cone $C$. Then, $C$ is the unique $k$-dimensional cone containing $\mathbf{r}(I)$.

Proof. Let $D$ be any other $k$-dimensional cone on which $\mathbf{r}$ is a geodesic. We want to show that $D=C$. First suppose the case $k=2$ has been proved. By Theorem $6.1, \mathbf{r}$ is a non-colliding geodesic on a 2-dimensional cone $\tilde{C} \subseteq C$ of the same aperture as $C$, and also on a 2-dimensional cone $\tilde{D} \subseteq D$ of the same aperture as $D$. Hence, $\tilde{C}=\tilde{D}$, and this cone has the same aperture $\psi$ as $C$ and $D$. Proposition 6.3 shows that $C$ is the unique cone of aperture $\psi$ containing $\tilde{C}=\tilde{D}$, and the same is true for $D$, so we have $C=D$. We may thus assume that $C$ and $D$ are 2-dimensional.

Now, since $\mathbf{r}$ is non-colliding, we can find three points in $\mathbf{r}(I) \subseteq C$ such that no two of them are collinear with the origin. Using Proposition 6.1, we find that the radial projection of these points on the unit sphere gives 3 distinct points on a circle. Hence, we get 3 noncollinear points on the 2-dimensional affine plane $P$ generating $C$. Now, the 2-dimensional affine plane $Q$ generating $D$ must also contain these 3 non-collinear points. Since an affine 2-dimensional plane in $\mathbb{R}^{n}$ is uniquely defined by 3 non-collinear points, we have $Q=P$, and hence $D=C$. 


\section{Particle motion in Yang's monopole and geodesics on cones}

In this section we investigate the solutions to the Poincaré problem in $\dot{\mathbb{R}}^{5}$. That is, we solve the equations (5.1), (5.2) and (5.3) for the motion of a charged particle in the field Yang's SU(2) monopole at the origin of Euclidean space $\mathbb{R}^{5}$.

Let us denote a solution to the Poincaré problem in $\dot{\mathbb{R}}^{5}$ by a pair $(\mathbf{r}, \mathbf{e})$ of curves $\mathbf{r}: I \rightarrow \dot{\mathbb{R}}^{5}$ and $\mathbf{e}: I \rightarrow \mathbb{R}^{3}$, for some open interval $I$. More precisely, $(\mathbf{r}, \mathbf{e})$ is a solution if the curve $(\mathbf{u}, r): I \rightarrow \mathbb{R}^{4} \times \mathbb{R}^{+}$obtained by expressing $\mathbf{r}$ in the stereographic projection coordinates from the north pole (4.5) together with the curve $\mathbf{e}=\left(e^{1}, e^{2}, e^{2}\right): I \rightarrow \mathbb{R}^{3}$ satisfy the equations of motion (5.1), (5.2) and (5.3) for all $t \in I$.

Our main goal is to show that for every solution $(\mathbf{r}, \mathbf{e})$ there is a 4-dimensional cone with vertex at the origin of $\mathbb{R}^{5}$ on which $\mathbf{r}$ is a geodesic. Note that this fact together with Theorem 6.1 show that $\mathbf{r}$ is also a geodesic on a 2-dimensional cone, as was the case for every solution to the Poincaré problem in $\dot{\mathbb{R}}^{3}$. It is quite remarkable that although we are dealing with a non-Abelian monopole and hence far more intricate equations of motion, the space of solution is almost identical to the one describing motion of a particle in the simpler Abelian Dirac monopole.

For Dirac's monopole, the hard part of the proof is to find an explicit expression for the direction $\mathbf{L} \in \dot{\mathbb{R}}^{3}$ of the cone. Once we have $\mathbf{L}$, it is very easy to see that $\mathbf{r}$ is at a constant angle from $\mathbf{L}$ and that $\ddot{\mathbf{r}}$ is always normal to the surface of the cone. For Yang's monopole, we will also find a vector $\mathbf{L} \in \dot{\mathbb{R}}^{5}$ for which $\mathbf{r}$ is at a constant angle, which will then imply that $\mathbf{r}$ lies on a 4-dimensional cone $C$. However, the proof that $\mathbf{r}$ is a geodesic on $C$ is more tricky. We will show that $\ddot{\mathbf{r}}$ is orthogonal to $\dot{\mathbf{r}}$ and $\mathbf{r}$, as we did for Dirac's monopole, but in $\mathbb{R}^{5}$ this fact is not sufficient to infer that $\ddot{\mathbf{r}}$ is normal to the surface of the cone. To complete the proof we will need to use some non-trivial conclusions of the preceding section on higher dimensional cones, namely, Corollaries 6.1 and 6.2.

Now, looking at the equations of motion (5.1), (5.2) and (5.3) for the Poincaré problem in $\dot{\mathbb{R}}^{5}$, we immediately see that a colliding curve is a solution if and only if it has constant speed, as was the case for Dirac's monopole. Since a constant-speed colliding curve is a geodesic of many cones, we may exclude these trivial solutions from our discussion. The main result of our paper is the following.

Theorem 7.1. Let $(\mathbf{r}, \mathbf{e})$ be a solution to the Poincaré problem in $\dot{\mathbb{R}}^{5}$. If $\mathbf{r}$ is non-colliding, then $\mathbf{r}$ is a geodesic on the 4-dimensional cone directed along the constant vector

$$
\mathbf{L}:=\left(\frac{\left(|\mathbf{e}|^{2}-4 r^{2}(\mathbf{A} \dot{\mathbf{u}} \cdot \mathbf{e})\right) \mathbf{u}+2 r^{2} \mathbf{E} \dot{\mathbf{u}}}{2\left(|\mathbf{u}|^{2}+1\right)}, \frac{2 r^{2}(\mathbf{A} \dot{\mathbf{u}} \cdot \mathbf{e})}{|\mathbf{u}|^{2}+1}+\frac{|\mathbf{e}|^{2}}{4} \frac{|\mathbf{u}|^{2}-1}{|\mathbf{u}|^{2}+1}\right) \in \mathbb{R}^{5}
$$

and of aperture $\psi$ given by

$$
\cos \psi=\frac{|\mathbf{e}|}{2}\left(\frac{|\mathbf{e}|^{2}}{4}+\frac{4 r^{4}|\dot{\mathbf{u}}|^{2}}{\left(|\mathbf{u}|^{2}+1\right)^{2}}\right)^{-1 / 2} .
$$

Proof. We first show that $\mathbf{r}$ is a geodesic on some 4-dimensional cone. By Corollary 6.1, it suffices to show that $\mathbf{r}$ lies on a 2 -dimensional cone and $\ddot{\mathbf{r}}$ is orthogonal to $\mathbf{r}$ and $\dot{\mathbf{r}}$. In the stereographic projection coordinates from the north pole $(\mathbf{u}, r)=\left(u_{1}, \ldots, u_{4}, r\right)$, we have

$$
\mathbf{r}=\left(\frac{2 r \mathbf{u}}{|\mathbf{u}|^{2}+1}, r \frac{|\mathbf{u}|^{2}-1}{|\mathbf{u}|^{2}+1}\right)
$$

and a straightforward computation shows that

$$
\ddot{\mathbf{r}} \cdot \mathbf{r}=\ddot{r} r-\frac{4 r^{2}|\dot{\mathbf{u}}|^{2}}{\left(|\mathbf{u}|^{2}+1\right)^{2}}, \quad \ddot{\mathbf{r}} \cdot \dot{\mathbf{r}}=\ddot{r} \dot{r}+4 \frac{\dot{r} r|\dot{\mathbf{u}}|^{2}+r^{2} \dot{\mathbf{u}} \cdot \ddot{\mathbf{u}}}{\left(|\mathbf{u}|^{2}+1\right)^{2}}-8 \frac{r^{2}|\dot{\mathbf{u}}|^{2}(\mathbf{u} \cdot \dot{\mathbf{u}})}{\left(|\mathbf{u}|^{2}+1\right)^{3}} .
$$


By inserting the equations of motion (5.1) and (5.2), we immediately get that these two expressions are equal to zero.

Now, to show that $\mathbf{r}$ lies on a 2-dimensional cone, we will follow an approach very similar to the one in the proof of Theorem 6.1. That is, let $\alpha: I \rightarrow \mathbb{R}^{5}$ be defined by $\alpha(t):=\mathbf{r}(t) /|\mathbf{r}(t)|$. It suffices to show that $\alpha(I)$ is contained in a 2-dimensional affine plane, or equivalently, that $\{\dot{\alpha}, \ddot{\alpha}\}$ is everywhere linearly independent while $\{\dot{\alpha}, \ddot{\alpha}, \dddot{\alpha}\}$ is everywhere linearly dependent. The proof that $\{\dot{\alpha}, \ddot{\alpha}\}$ is everywhere linearly independent is exactly the same as the one in the proof of Theorem 6.1. Now, we will show an explicit non-trivial linear dependence of $\{\dot{\alpha}, \ddot{\alpha}, \ddot{\alpha}\}$. First, we have

$$
\alpha=\left(\frac{2 \mathbf{u}}{|\mathbf{u}|^{2}+1}, \frac{|\mathbf{u}|^{2}-1}{|\mathbf{u}|^{2}+1}\right), \quad \dot{\alpha}=\left(\frac{2 \dot{\mathbf{u}}}{|\mathbf{u}|^{2}+1}-\frac{4(\mathbf{u} \cdot \dot{\mathbf{u}}) \mathbf{u}}{\left(|\mathbf{u}|^{2}+1\right)^{2}}, \frac{4 \mathbf{u} \cdot \dot{\mathbf{u}}}{\left(|\mathbf{u}|^{2}+1\right)^{2}}\right) .
$$

We then compute the second and third derivatives of $\alpha$ by inserting the equations of motion (5.1), (5.2) and (5.3) to eliminate all second and higher derivatives of $(\mathbf{u}, r)$. We find

$$
\begin{aligned}
\ddot{\alpha}= & \left(\frac{\mathbf{E} \dot{\mathbf{u}}-2(\mathbf{A} \dot{\mathbf{u}} \cdot \mathbf{e}) \mathbf{u}}{r^{2}\left(|\mathbf{u}|^{2}+1\right)}-\frac{8|\dot{\mathbf{u}}|^{2} \mathbf{u}}{\left(|\mathbf{u}|^{2}+1\right)^{3}}+\frac{4 \dot{r}}{r}\left(\frac{2(\mathbf{u} \cdot \dot{\mathbf{u}}) \mathbf{u}}{\left(|\mathbf{u}|^{2}+1\right)^{2}}-\frac{\dot{\mathbf{u}}}{|\mathbf{u}|^{2}+1}\right),\right. \\
& \left.\frac{2 \mathbf{A} \dot{\mathbf{u}} \cdot \mathbf{e}}{r^{2}\left(|\mathbf{u}|^{2}+1\right)}-\frac{4|\dot{\mathbf{u}}|^{2}\left(|\mathbf{u}|^{2}-1\right)}{\left(|\mathbf{u}|^{2}+1\right)^{3}}-\frac{8 \dot{r} \mathbf{u} \cdot \dot{\mathbf{u}}}{r\left(|\mathbf{u}|^{2}+1\right)^{2}}\right), \\
\dddot{\alpha}= & \left(\frac{12 r \dot{r}(2(\mathbf{A} \dot{\mathbf{u}} \cdot \mathbf{e}) \mathbf{u}-\mathbf{E} \dot{\mathbf{u}})-|\mathbf{e}|^{2} \dot{\mathbf{u}}}{2 r^{4}\left(|\mathbf{u}|^{2}+1\right)}+\frac{|\mathbf{e}|^{2}(\mathbf{u} \cdot \dot{\mathbf{u}}) \mathbf{u}}{r^{4}\left(|\mathbf{u}|^{2}+1\right)^{2}}+\frac{24|\dot{\mathbf{u}}|^{2}(2 \dot{r} \mathbf{u}-r \dot{\mathbf{u}})}{r\left(|\mathbf{u}|^{2}+1\right)^{3}}\right. \\
& +\frac{48|\dot{\mathbf{u}}|^{2}(\mathbf{u} \cdot \dot{\mathbf{u}}) \mathbf{u}}{\left(|\mathbf{u}|^{2}+1\right)^{4}}+\frac{12 \dot{r}^{2}}{r^{2}}\left(\frac{\dot{\mathbf{u}}}{|\mathbf{u}|^{2}+1}-\frac{2(\mathbf{u} \cdot \dot{\mathbf{u}}) \mathbf{u}}{\left(|\mathbf{u}|^{2}+1\right)^{2}}\right),-\frac{12 \dot{r} \mathbf{A} \dot{\mathbf{u}} \cdot \mathbf{e}}{r^{3}\left(|\mathbf{u}|^{2}+1\right)} \\
& \left.-\frac{|\mathbf{e}|^{2} \mathbf{u} \cdot \dot{\mathbf{u}}}{r^{4}\left(|\mathbf{u}|^{2}+1\right)^{2}}+\frac{24 \dot{r}|\dot{\mathbf{u}}|^{2}\left(|\mathbf{u}|^{2}-1\right)}{r\left(|\mathbf{u}|^{2}+1\right)^{3}}-\frac{48|\mathbf{u}|^{2} \mathbf{u} \cdot \dot{\mathbf{u}}}{\left(|\mathbf{u}|^{2}+1\right)^{4}}+\frac{24 \dot{r}^{2} \mathbf{u} \cdot \dot{\mathbf{u}}}{r^{2}\left(|\mathbf{u}|^{2}+1\right)^{2}}\right) .
\end{aligned}
$$

To perform these computations, it is useful to first derive the following identities

$$
\begin{aligned}
& \mathbf{u} \cdot \mathbf{E} \dot{\mathbf{u}}=\left(|\mathbf{u}|^{2}+1\right) \mathbf{A} \dot{\mathbf{u}} \cdot \mathbf{e}, \\
& \mathbf{e A E}=-\frac{|\mathbf{e}|^{2} \mathbf{u}}{|\mathbf{u}|^{2}+1}, \\
& \dot{\mathbf{E}} \dot{\mathbf{u}}=2 \frac{|\dot{\mathbf{u}}|^{2} \mathbf{E} \mathbf{u}+(\mathbf{u} \cdot \mathbf{E} \dot{\mathbf{u}}) \dot{\mathbf{u}}-(\mathbf{u} \cdot \dot{\mathbf{u}}) \mathbf{E} \dot{\mathbf{u}}}{|\mathbf{u}|^{2}+1} .
\end{aligned}
$$

We then get the simple expression

$$
\left(\frac{|\mathbf{e}|^{2}}{4 r^{2}}+\frac{6 \dot{r}^{2}}{r^{2}}+\frac{12|\dot{\mathbf{u}}|^{2}}{\left(|\mathbf{u}|^{2}+1\right)^{2}}\right) \dot{\alpha}+\frac{6 \dot{r}}{r} \ddot{\alpha}+\dddot{\alpha}=0 .
$$

Hence, $\{\dot{\alpha}, \ddot{\alpha}, \dddot{\alpha}\}$ is everywhere linearly dependent, so $\mathbf{r}$ lies on a 2-dimensional cone, and by Corollary $6.1, \mathbf{r}$ is a geodesic on a 4 -dimensional cone $C$.

We will now give an explicit expression for the cone $C$. By differentiating the vector $\mathbf{L}$ given by (7.1) and inserting the equations of motion, we see that $\mathbf{L}$ is constant. Moreover,

$$
\frac{\mathbf{r} \cdot \mathbf{L}}{|\mathbf{r}||\mathbf{L}|}=\frac{|\mathbf{e}|^{2}}{4|\mathbf{L}|}=\frac{|\mathbf{e}|}{2}\left(\frac{|\mathbf{e}|^{2}}{4}+\frac{4 r^{4}|\dot{\mathbf{u}}|^{2}}{\left(|\mathbf{u}|^{2}+1\right)^{2}}\right)^{-1 / 2},
$$

which is also constant. Since $\mathbf{r}$ is non-colliding we have $\dot{\mathbf{u}} \neq 0$, so this expression can be written as the cosine of some angle $\psi \in(0, \pi / 2]$. Hence, $\mathbf{r}$ lies on the 4-dimensional cone of aperture $\psi$ directed along $\mathbf{L}$. But we showed that $\mathbf{r}$ is a geodesic on some 4-dimensional cone $C$, and by Corollary 6.2, $C$ is the unique 4-dimensional cone containing $\mathbf{r}(I)$. Hence, $C$ is the cone of aperture $\psi$ directed along $\mathbf{L}$. 
By this theorem, the problem reduces to the geodesic equations on a 4-dimensional cone, which in turn reduces to the geodesic equations on a 2-dimensional cone by Theorem 6.1. Geodesics on 2-dimensional cones were discussed in Section 2.

\section{Acknowledgements}

The author is grateful to Professor Niky Kamran for his constant guidance and invaluable suggestions. The author would also like to thank the anonymous referees who provided helpful comments, corrections and reference suggestions. This work was supported by the NSERC USRA program, grant number RGPIN 105490-2011.

\section{References}

[1] Bai Z., Meng G., Wang E., On the orbits of magnetized Kepler problems in dimension $2 k+1, J$. Geom. Phys. 73 (2013), 260-269, arXiv:1302.7271.

[2] Cho Y.M., Higher-dimensional unifications of gravitation and gauge theories, J. Math. Phys. 16 (1975), 2029-2035.

[3] Dirac P.A.M., Quantised singularities in the electromagnetic field, Proc. R. Soc. Lond. Ser. A 133 (1931), 60-72.

[4] Duval C., Horváthy P., Particles with internal structure: the geometry of classical motions and conservation laws, Ann. Physics 142 (1982), 10-33.

[5] Fehér L.G., The $\mathrm{O}(3,1)$ symmetry problem of the charge-monopole interaction, J. Math. Phys. 28 (1987), 234-239.

[6] Fierz M., Zur Theorie magnetisch geladener Teilchen, Helvetica Phys. Acta 17 (1944), 27-34.

[7] Goddard P., Olive D.I., Magnetic monopoles in gauge field-theories, Rep. Progr. Phys. 41 (1978), $1357-1437$.

[8] Haas F., Noether symmetries for charged particle motion under a magnetic monopole and general electric fields, physics/0211074.

[9] Harnad J., Paré J.P., Kaluza-Klein approach to the motion of nonabelian charged particles with spin, Classical Quantum Gravity 8 (1991), 1427-1444.

[10] Horváthy P.A., The dynamical symmetries of the monopole in geometric quantization, Lett. Math. Phys. 7 (1983), 353-361.

[11] Iwai T., The geometry of the SU(2) Kepler problem, J. Geom. Phys. 7 (1990), 507-535.

[12] Jackiw R., Dynamical symmetry of the magnetic monopole, Ann. Physics 129 (1980), 183-200.

[13] Kerner R., Generalization of the Kaluza-Klein theory for an arbitrary non-abelian gauge group, Ann. Inst. H. Poincaré Sect. A 9 (1968), 143-152.

[14] Lapidus I.R., Pietenpol J.L., Classical interaction of an electric charge with a magnetic monopole., Amer. J. Phys. 28 (1960), 17-18.

[15] McIntosh H.V., Cisneros A., Degeneracy in the presence of a magnetic monopole, J. Math. Phys. 11 (1970), 896-916.

[16] Meng G., Dirac and Yang monopoles revisited, Cent. Eur. J. Phys. 5 (2007), 570-575, math-ph/0409051.

[17] Meng G., MICZ-Kepler problems in all dimensions, J. Math. Phys. 48 (2007), 032105, 14 pages, math-ph/0507028.

[18] Meng G., The Poisson realization of $\mathfrak{s o}(2,2 k+2)$ on magnetic leaves and generalized MICZ-Kepler problems, J. Math. Phys. 54 (2013), 052902, 14 pages, arXiv:1211.5992.

[19] Minami M., Dirac's monopole and the Hopf map, Progr. Theoret. Phys. 62 (1979), 1128-1142.

[20] Minami M., Quaternionic gauge-fields on $S^{7}$ and Yang's SU(2) monopole, Progr. Theoret. Phys. 63 (1980), 303-321.

[21] Montgomery R., Canonical formulations of a classical particle in a Yang-Mills field and Wong's equations, Lett. Math. Phys. 8 (1984), 59-67.

[22] Montgomery R., MICZ-Kepler: dynamics on the cone over SO(n), Regul. Chaotic Dyn. 18 (2013), 600-607, arXiv:1305.1063. 
[23] Moreira I.C., Ritter O.M., Santos F.C., Lie symmetries for the charge-monopole problem, J. Phys. A: Math. Gen. 18 (1985), L427-L430.

[24] Orzalesi C.A., Pauri M., Geodesic motion in multidimensional unified gauge theories, Nuovo Cimento B 68 (1982), 193-202.

[25] Poincaré H., Remarques sur une expérience de M. Birkeland, Compt. Rend. Acad. Sci. Paris 123 (1896), 530-533.

[26] Ritter O.M., Symmetries and invariants for some cases involving charged particles and general electromagnetic fields: a brief review, Braz. J. Phys. 30 (2000), 438-454.

[27] Ryder L.H., Dirac monopoles and the Hopf map $S^{3} \rightarrow S^{2}$, J. Phys. A: Math. Gen. 13 (1980), $437-447$.

[28] Sivardière J., On the classical motion of a charge in the field of a magnetic monopole, Eur. J. Phys. 21 (2000), 183-190.

[29] Spivak M., A comprehensive introduction to differential geometry, Vol. 4, Publish or Perish, Boston, Mass., 1975.

[30] Sternberg S., Minimal coupling and the symplectic mechanics of a classical particle in the presence of a Yang-Mills field, Proc. Nat. Acad. Sci. USA 74 (1977), 5253-5254.

[31] Trautman A., Solutions of Maxwell and Yang-Mills equations associated with Hopf fibrings, Internat. J. Theoret. Phys. 16 (1977), 561-565.

[32] Weinstein A., A universal phase space for particles in Yang-Mills fields, Lett. Math. Phys. 2 (1978), 417-420.

[33] Wong S.K., Field and particle equations for the classical Yang-Mills field and particles with isotopic spin, Nuovo Cimento A 65 (1970), 689-694.

[34] Yang C.N., Generalization of Dirac's monopole to $\mathrm{SU}_{2}$ gauge fields, J. Math. Phys. 19 (1978), 320-328.

[35] Zwanziger D., Exactly soluble nonrelativistic model of particles with both electric and magnetic charges, Phys. Rev. 176 (1968), 1480-1488. 